\title{
Residual Stresses in Cast Iron Components - Simulated Results Verified by Experimental Measurements
}

\author{
Lennart Elmquist ${ }^{1, a^{*}}$, Aron Brehmer ${ }^{1, b}$, Pål Schmidt $^{2, c}$ and Björn Israelsson ${ }^{3, d}$ \\ ${ }^{1}$ Swerea SWECAST, P.O. Box 2033, SE-550 02 Jönköping, Sweden \\ ${ }^{2}$ Volvo GTT, 40508 Göteborg, Sweden \\ ${ }^{3}$ SKF Mekan AB, P.O. Box 89, 64121 Katrineholm, Sweden

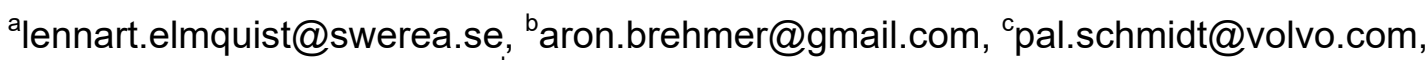 \\ dbjorn.israelsson@skf.com
}

Keywords: Residual stresses, hole-drilling, sectioning, simulation, deformations, strains, cast iron.

\begin{abstract}
The aim of this work was to investigate how accurately residual stresses can be simulated in a cast component. The reason why this is important is that a simulation that also considers residual stresses can be used to design cast components more weight- and cost-efficient. The verification was done by comparing simulated results with physical measurements on cylinders made of ductile iron. The measurements were performed with two methods, sectioning and holedrilling. These methods were applied on both as-cast and machined cylinders. The simulations were based on process data from casting trials and material data from the simulation software database. The material data for the heat conductivity of the molding sand was fitted to get a good conformity between measured and simulated temperatures. This was done to ensure that the residual stress simulation used a relevant temperature history. It turned out that the simulation was in good agreement with results from the sectioning measurements of the axial stress while results from the hole-drilling measurements were contradictive. These results were contradictive for both as-cast and machined cylinders. It can therefore be concluded that residual stresses measured by the sectioning method resulted in good conformity with the simulated $\sigma_{\mathrm{z}}$ stresses. It was also concluded that the feeders, acting as extra heat sources, affects residual stresses locally and contributes to differences in stresses beneath the feeders, compared to corresponding areas between the feeders. It was also found that the resolution of the mesh needs to be finer to take into account changes in the stresses with increments in depth when using the hole drilling method.
\end{abstract}

\section{Introduction}

Residual stresses are those stresses that remain in a component after the external load has been removed. These stresses are present in the absence of any external forces and/or thermal gradients. This means that when a load is applied, the different stresses will be added on top of each other, and if the residual stresses are tensile the maximum stress level might be higher than expected and the maximum tolerable level exceeded. Residual stresses may lead to warping, distortion, or cracking. There are different sources for residual stresses and they are classified as mechanical, thermal, or chemical. Mechanical is for example due to manufacturing processes that produce non-uniform plastic deformation. Thermally induced stresses on a macroscopic level arise due to non-uniform heating or cooling and on a microscopic level as a consequence of different CTE (coefficient of thermal expansion) between different phases and constituents. Chemically generated stresses develop due to different volume changes associated with for example chemical reactions [1]. Castings are exposed to both external and internal constraints during solidification and cooling. This may lead to unevenly distributed strains and consequently residual stresses. External constraints depend on the shape of the casting and how much the material can contract and redistribute during solidification and cooling. This is also related to the properties of the mold. Internal constraints depend on differences in cooling rate in different parts of the casting leading to 
uneven contractions. Also the cooling after solidification and the solid state transformation cause differences in contraction.

Residual stresses are often classified based on the length scale they are acting on [1,2]:

- Type I (macro), a scale larger than the grain size of the material.

- Type II (micro/meso), residual stresses that vary on the scale of individual grains.

- Type III (micro), within a single grain due to e.g. dislocations or other crystalline defects.

It is important to know whether the stresses are tensile or compressive as the difference is of paramount meaning. Tensile stresses at the surface increase the risk for nucleation and propagation of cracks. Compressive stresses on the other hand have the opposite effect and counteract the formation of cracks, and compressive stresses are therefore sometimes intentionally introduced to the surface by for example shot peening.

Even if it is common to talk about residual stress measurements, it is not stresses that are measured but instead deformations and strains. Residual stresses are then calculated based on these measured strains. This means that the residual stresses obtained also depends on the choice of method and equations for the calculation, and also the material data used in these calculations. There are different methods to measure residual stresses. They are defined as mechanical or physical based on the principle they are based on and sometimes also classified as destructive, nondestructive or semi-destructive depending on how much the component is affected by the measurement. In most cases the choice of method depends on the scale of the structure that is to be measured [1,2].

Sectioning. This is a destructive method as the stresses are released by cutting through the component. The strains are measured by strain gauges attached close to the cut. It is important that the cutting does not produce any new stresses in the material, as these stresses would also be measured and influence the result. During sectioning strains are measured in only one direction. The method is therefore best suited for measurements where the stress is known to be in one direction. It is also assumed that the stresses are equal on the cutting depth influencing the strain gauges. By placing strain gauges on both sides of the component and cut all the way through the component a stress variation profile can be estimated.

Hole-drilling. This method is based on the deformations formed due to stresses released when a hole is drilled. The strains are measured around the drilled hole and near the surface, with a depth of only a few millimeters. The method is defined as semi-destructive as the hole often is so small it can be repaired or neglected [3]. Strain gauges are attached around the hole, their number and configuration are selected depending on the expected results and the geometry where the measurement is to be done. The procedure starts by locating the zero-point on the surface. As the hole is drilled stresses are released and the corresponding strains are measured. The residual stresses are then calculated based on the measured strains. Hole-drilling is limited to stress states below about $60 \%$ of the yield strength [4]. The drilling is usually done stepwise as it gives a better picture of the stress state if not equal over drill depth $[5,6]$. It is important to avoid any formation of plastic deformations from the drilling as this would interfere with the measurements. Therefore high speed drills or abrasive air jets are used. Hole-drilling is the only method so far accepted as an ASTM standard [7].

The aim of the present project was to develop more cost-efficient castings by taking also residual stresses into account. Today the designer often assumes the material is free from stresses and that the properties are homogeneous when the casting is designed. If simulations can be used to correctly predict where the residual stresses will appear, if being tensile or compressive, and also their magnitude, and at the same time also consider local variations in material properties, the design can be more precise and correct. This will in turn reduce weight and cost.

Investigations have shown that as much as $80 \%$ of the residual stresses can be eliminated by heat treatment [8]. With a better understanding of these stresses and the possibility to better predict them, the energy consuming and costly heat treatment can be optimized, or even eliminated. 


\section{Experimental Procedure}

Casting experiment. Seven cylinders were cast in sand molds made of Alphaset. No cores were used. The whole cylinder was formed in the drag and to each cylinder there are three feeders placed in the cope. In each mold there was also a second cylinder, however, this cylinder was only used for production purposes and not included in the experiment. The cylinders with feeders and the gating system used in the simulations are shown in Fig 1. There are two ingates to fill each cylinder.
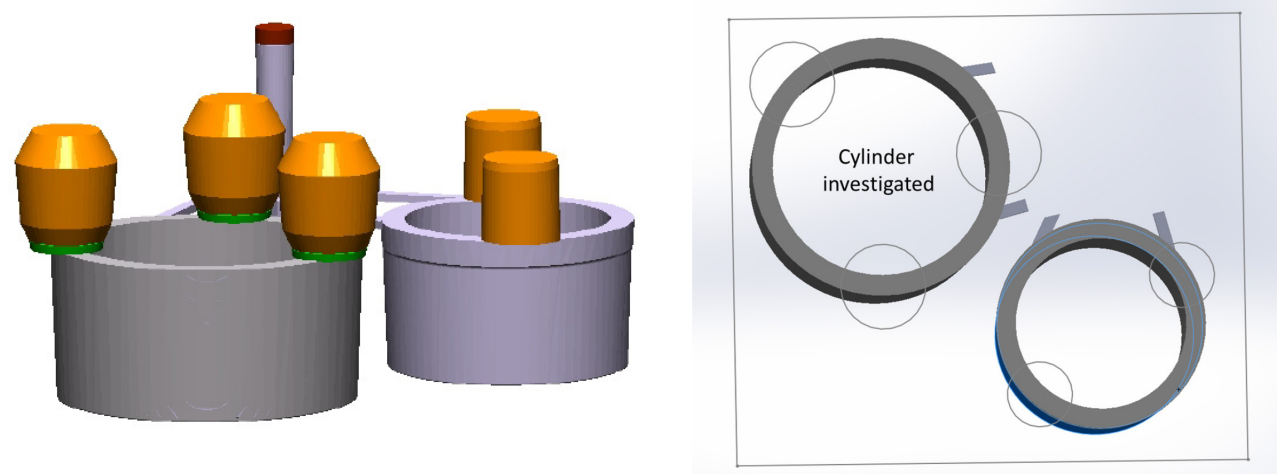

Figure 1. Geometry of the cylinders with feeders and gating system. The smaller cylinder to the right is not included in the investigation.

The cast iron used was a ductile iron, GJS 500-7, from two different nodularizing treatments, but from the same base iron. Chemical analysis was done on iron from both treatments. After shake out, three of the cylinders were used to measure residual stresses in as-cast condition while three of the cylinders were machined before measurements. The machining consisted of turning of both inner and outer surfaces of the cylinders. The seventh cylinder was used only to measure the temperatures during solidification and cooling. The temperatures were measured using six thermocouples type K with a diameter of $3 \mathrm{~mm}$. Four of these thermocouples were positioned in the casting, and the other two were positioned in the sand mold, see Fig 2. Two of the thermocouples in the casting, No 1 and 4 , were protected using a ceramic tube while the other two, No 2 and 3, were unprotected during measurement. The result from these measurements was compared with the simulated temperatures.

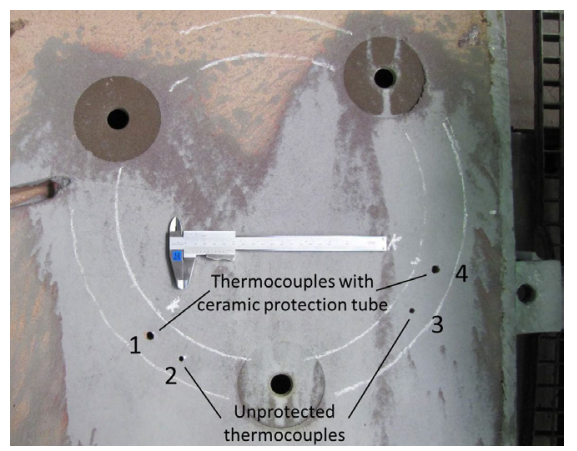

Figure 2. Position of the thermocouples shown in the cope part of the mold.

The residual stresses were measured using two methods, sectioning and hole-drilling. Holedrilling was done on two as-cast samples and two machined samples while sectioning was done on one sample of each.

Sectioning. Measurements were done using axial strain gauges attached to both the inner and outer surfaces of the cylinder, see Fig 3a. Strain gauges on the inner surface have numbers 1-2 and those on the outer surface are numbered 3-4. The strain gauges were of type KFG-5-120-C111L3M3R from Kyowa. In order to release the stresses, three cuts were made, see Fig $3 b$. The cutting is supposed to not totally eliminate all stresses but leave some MPa left. By simulating the cutting in MAGMAsoft as a machining operation the measured and simulated values were compared. 
a)

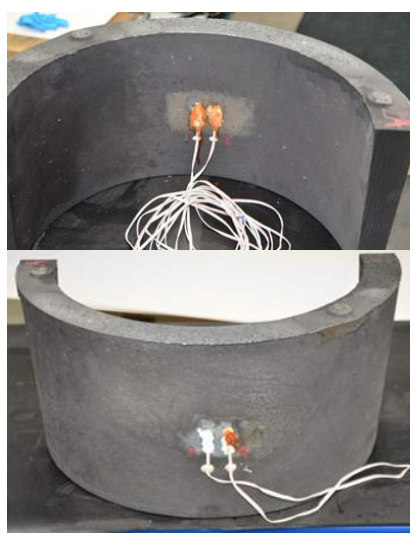

b)

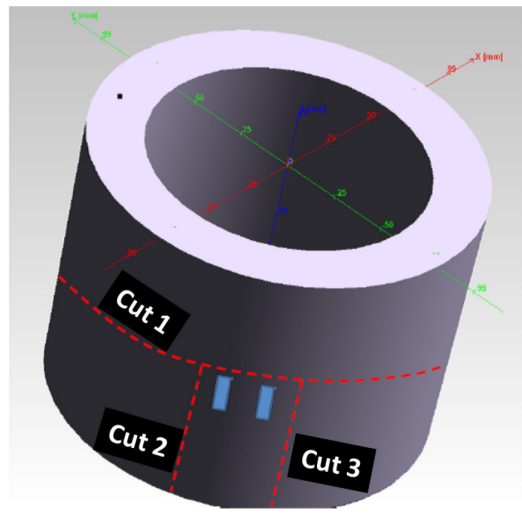

Figure 3. a) Position of the strain gauges during sectioning, b) the different cuts.

Hole-drilling. The surface of the as-cast components was prepared before attachment of the strain gauges. This was done by first brushing away all residual molding sand followed by grinding using P240 grinding paper to get a surface flat enough to attach the strain gauges. The surface was then washed with ethanol followed by cleaning with a mixture of isopropanol and acetone (RMS1 from HBM). Also the machined samples were cleaned and washed to facilitate mounting of the strain gauges. The strain gauges used were 1.5/120R RY61 from HBM. These were glued to the cylinders with cold-hardening cyanoacrylate glue. On each cylinder two measurements were done and this was done on two positions approximately opposite to each other. The equipment used to measure the strains was MTS 3000-RESTAN from Sint technology. This system is based on high speed drilling using an air-turbine with 350000-400000 rpm. It is equipped with automatic incremental drilling with a step engine. The zero-point is found by the aid of electrical contact between the drill and the metal surface. The parameters used during the measurements are summarized in Table 1.

Table 1. Parameters used for hole-drilling.

\begin{tabular}{llll}
\hline Parameter & Value & Parameter & Value \\
\hline Drill diameter & $1.6 \mathrm{~mm}$ & Waiting time drilling & $4 \mathrm{~s}$ \\
Number of steps & 40 & Waiting time before measurement & $5 \mathrm{~s}$ \\
Depth of each step & $0.04 \mathrm{~mm}$ & E-modulus & $169 \mathrm{GPa}$ \\
Drill speed & $20 \mathrm{~mm} / \mathrm{s}$ & Poisson's ratio & 0.27 \\
\hline
\end{tabular}

The analysis was made using the software EVAL v6.0. This software analyzes the measurements according to ASTM-E837 for non-uniform stress states.

Simulation. The simulation was done with MAGMAsoft version 5.3 and the modules MAGMAiron and MAGMAstress. A base simulation for calibration was created based on parameters from the casting trial and the software database. A simplified downsprue was used in the simulation and the molding material selected was furan-mold from the software database. In the simulations a pouring temperature of $1320^{\circ} \mathrm{C}$ was used and the pouring time was $21 \mathrm{~s}$. The number of elements was 7.9 million in total and 480000 metal elements.

The first step was to calibrate the solidification and cooling times to secure that MAGMAstress was fed with the relevant temperature history. The parameters used to calibrate the simulation to the measured results were inoculation, mold sand conductivity and the heat transfer coefficient.

The residual stresses were calculated using MAGMAstress. The calculation was done until the maximum temperature in the cylinder was $200^{\circ} \mathrm{C}$. Temperature dependent mechanical properties for the metal were selected from the software database for GJS 500-7. In these simulations only a part of the mold around the cylinder was included. Three different simulations were compared. In two of them the mold was assumed to affect the residual stresses and contact conditions was calculated between the mold and casting. The difference between them was the stiffness of the mold. The first version (V1) used data from the software database. In the second version (V2) the 
Young's modulus for the furan sand was lowered from $1500 \mathrm{MPa}$ to $250 \mathrm{MPa}$. The value $250 \mathrm{MPa}$ was found in the literature and considered to be interesting to investigate as it deviated substantially from the value in the database [9]. In the third version (V3) it was assumed that the sand mold does not affect the development of residual stresses. In these variants, residual stresses from the feeders were not calculated which means that these only affect the residual stresses by heat distribution and not any mechanical boundary conditions. All versions included a machining step in the simulation to take also the turning of the inner and outer surfaces of the cylinders into account. These three versions were then compared with the results from residual stress measurements.

\section{Results and Discussion}

Casting trial. The chemical composition in the two ladles is shown in Table 2. The filling time was measured when filling one of the molds and was found to be $21 \mathrm{~s}$. This time was then used in the simulations. The temperatures in both ladles were measured before filling the first molds. The temperature was $1339^{\circ} \mathrm{C}$ in Ladle 1 , and $1357^{\circ} \mathrm{C}$ in Ladle 2 . The mold with the thermocouples was the last mold filled from Ladle 1.

Table 2. Chemical composition in the two ladles [wt \%].

\begin{tabular}{|c|c|c|c|c|c|c|c|c|c|c|c|c|}
\hline & C & $\mathrm{Si}$ & $\mathrm{Mn}$ & $\mathrm{P}$ & $\mathrm{S}$ & $\mathrm{Cr}$ & $\mathrm{Ni}$ & $\mathrm{Al}$ & $\mathrm{Cu}$ & Co & $\mathrm{Mg}$ & $\mathrm{N}$ \\
\hline Ladle 1 & 3.6 & 2.19 & 0.388 & 0.01 & 0.006 & 0.032 & 0.035 & 0.010 & 0.295 & 0.02 & 0.038 & 0.01 \\
\hline Ladle 2 & 3.6 & 2.18 & 0.396 & 0.01 & 0.006 & 0.032 & 0.037 & 0.011 & 0.262 & 0.02 & 0.040 & 0.01 \\
\hline
\end{tabular}

Sectioning. Measurements were done on both the inner and outer surfaces of the cylinders. The results are summarized in Table 3. The measurements give a value of strains and stresses in the zdirection which is in the same direction as the center axis of the cylinder. The stresses have been calculated based on the measured strains and a Young's modulus of $170 \mathrm{GPa}$.

Table 3. Results from sectioning. Strains and stresses in z-direction.

\begin{tabular}{|c|c|c|c|c|c|c|c|c|c|c|c|}
\hline \multicolumn{6}{|c|}{ As-cast condition } & \multicolumn{6}{|c|}{ Machined condition } \\
\hline \multicolumn{3}{|c|}{ Strains inside $[\mu \mathrm{m} / \mathrm{m}]$} & \multicolumn{3}{|c|}{ Strains outside $[\mu \mathrm{m} / \mathrm{m}]$} & \multicolumn{3}{|c|}{ Strains inside $[\mu \mathrm{m} / \mathrm{m}]$} & \multicolumn{3}{|c|}{ Strains outside $[\mu \mathrm{m} / \mathrm{m}]$} \\
\hline 1 & 2 & $\mathrm{x}$ & 3 & 4 & $\mathrm{X}$ & $1^{1}$ & 2 & $\mathrm{X}$ & 3 & 4 & $\mathrm{X}$ \\
\hline 81 & 85 & 83 & -134 & -135 & -134.5 & -13 & 67 & 67 & -66 & -50 & -58 \\
\hline \multicolumn{3}{|c|}{ Stresses inside [MPa] } & \multicolumn{3}{|c|}{ Stresses outside $[\mathrm{MPa}]$} & \multicolumn{3}{|c|}{ Stresses inside $[\mathrm{MPa}]$} & \multicolumn{3}{|c|}{ Stresses outside $[\mathrm{MPa}]$} \\
\hline 1 & 2 & $\mathrm{X}$ & 3 & 4 & $\mathrm{X}$ & $1^{2}$ & 2 & $\mathrm{x}$ & 3 & 4 & $\mathrm{X}$ \\
\hline 13.8 & 14.4 & 14.1 & -22.8 & -23.0 & -22.9 & -2.2 & 11 & 11 & -11 & -8.5 & -9.75 \\
\hline
\end{tabular}

${ }^{1,2}$ Gauge is not included in the mean value

The results from the sectioning are reasonable apart from gauge number 1 on the machined sample where the result indicates the gauge detached during measurement resulting in deviating result. Therefore, this result has not been used in the subsequent comparison with the simulation. One uncertainty in these measurements is that it is difficult to know whether the cutting has released all the stresses in the material or if some stresses still are present also after the cutting and thus not included in the measurement. A simulation of the sectioning was done to get an estimation of the level of stresses that can be expected to be released during cutting. This simulation was done with the same parameters as simulation V3. The compressive stress is reduced by approximately $22 \mathrm{MPa}$ at the first cut. At the other two cuts not much more happens. The simulation shows that the stresses totally relax on the inside of the cylinder but after sectioning there are still residual stresses on the outside. If not all stresses are released during sectioning the measurements will show a wrong value with a too low absolute value which is a risk in this case. Another important thing is that the measurements only give stresses in the z-direction, which in the present case was assumed to be the relevant direction for the measurement. Therefore the measurements do not give the whole picture of the stress conditions in the cylinder as any radial or circumferential stresses not are considered. 
Hole-drilling. The result from both as-cast and machined samples was contradictory. For the cylinders as-cast it was only one measurement that gave a reasonable stable result, see Fig. 4 . In this measurement $\sigma_{\min }$ was in the same direction as $\sigma_{\mathrm{z}}$ in the interval apart from the first point. This point is right at the surface and the measurement is affected by how the surface looks and how well it was prepared for the measurement. The results from the other measurements vary in a way that makes them less reasonable. For the machined cylinders the measurements did not give any unambiguous result and not a single measurement was particularly reasonable and realistic.
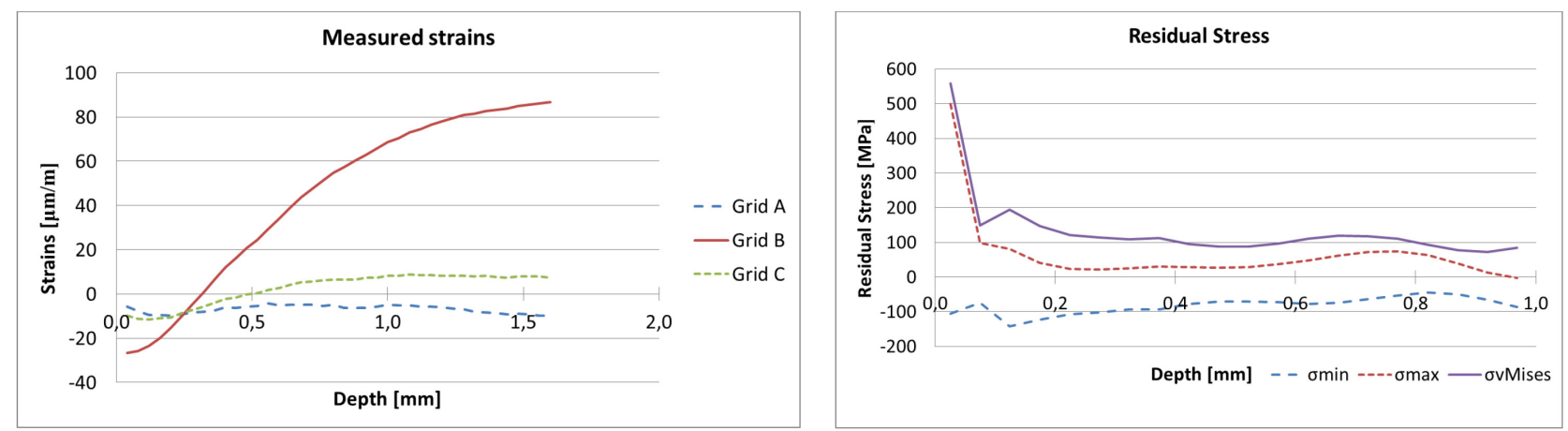

Figure 4. Measured strains and calculated stresses for as-cast measurement 1.

Two of the measurements, machined 1 and machined 4 showed similar curves for the direction of the stresses but gave different results for the stress levels. It seems like something has happened with the strain gauges when drilled a bit into the material on the two samples machined 2 and machined 3, as the measured strains in both cases jumps down in level at the same time and then continues to develop in the same direction.

Simulation of temperature history. This part was done to provide the residual stress simulation with relevant temperature history. Inoculation, mold sand conductivity and the heat transfer coefficient were varied to get a better conformity between the measured and simulated curves.

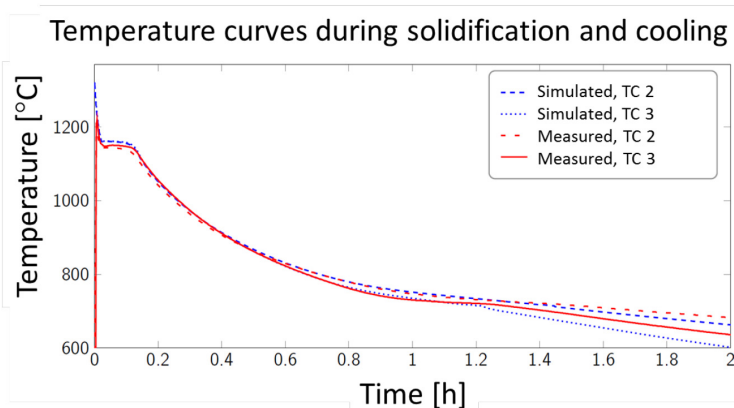

a)

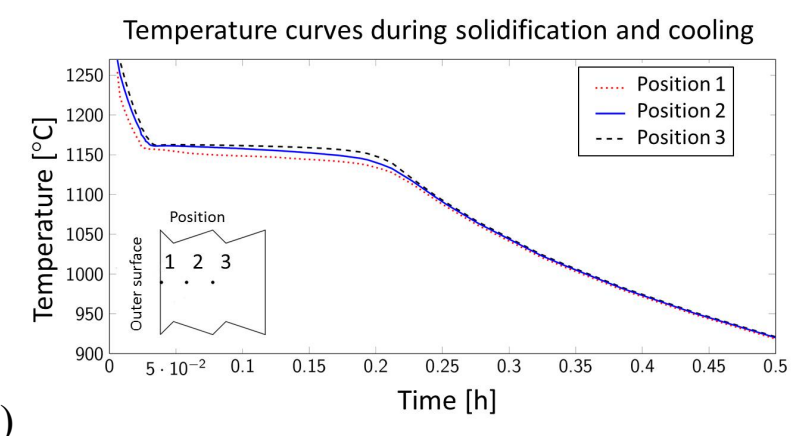

b)

Figure 5. a) Comparison between measured and simulated temperatures for thermocouples 2 and 3 , b) Simulated changes in temperature during solidification and cooling from center to surface of the cylinder.

In the final version only mold conductivity was changed as it also has the largest impact on the temperature history. A comparison between measured and simulated results for thermocouples 2 and 3 is shown in Fig 5a. The measured curves have reasonable solidification intervals and phase transformations as expected for this material. It is hard to measure the maximum temperature correctly during filling as the thermocouples have a certain response time and the peak for the maximum temperature is passed quickly. Besides, it is difficult to determine the exact position of the thermocouples inside the mold cavity. The most uncertain positioning is in the $\mathrm{x} / \mathrm{y}$-direction. An investigation of the difference in temperature radially from the simulation shows that this influences the temperature mostly during solidification and to lesser extent during the cooling, see Fig 5b. This difference could be a source of errors as subsequent comparison and adjustments of the simulation depends on the position of the thermocouples during measurement. 
Simulation of residual stresses. For an easier comparison between the simulations and the measurements a mean value of the simulated stresses in the same area as the measurements has been calculated. These results are shown in Table 4 together with the results from the sectioning. The values in Table 4 show that the simulated stresses in the area for sectioning is largest for V1 (rigid mold), smallest for V2 (weak mold) while V3 is in between (negligible mold). As the mold is restricting contractions of the casting during solidification this explains the highest stresses in the rigid mold (V1). With this motivation the stresses in V2 (weak mold) should be higher than those in V3 (negligible mold) as V2 still is holding back to some extent. The explanation to why this is not the case was not found during the work. As discussed earlier it can be assumed that not all stresses are released during sectioning and in that case there is a good agreement between the measurements and V1 and V3. With that argument V1 should agree better than V3 as the inner surface also is included. However, both measured and simulated values are close to each other for V1 and V3 which means it is hard to say which of the simulations that best fits, especially as only one measurement with two gauges per side was performed.

Table 4. Comparison of simulated and measured residual stresses.

\begin{tabular}{ccc|cc}
\hline & \multicolumn{2}{c|}{ As-cast condition } & \multicolumn{2}{c}{ Machined condition } \\
\hline Version & $\sigma_{\mathbf{z}}(\mathbf{M P a})$ outside & $\boldsymbol{\sigma}_{\mathbf{z}}(\mathbf{M P a})$ inside & $\boldsymbol{\sigma}_{\mathbf{z}}(\mathbf{M P a})$ outside & $\boldsymbol{\sigma}_{\mathbf{z}}(\mathbf{M P a})$ inside \\
\hline V1 (contact, rigid mold) & -31 & 18 & -14 & 14 \\
V2 (contact, weak mold) & -26 & 6 & -4 & 3 \\
V3 (no contact) & -32 & 12 & -8 & 7 \\
Sectioning & -22.9 & 14.1 & -9.75 & 11 \\
\hline
\end{tabular}

The influence of the feeders has also been investigated in the simulations. The residual stress profile through the cylinder wall on half the height of the cylinder is shown in Fig 6. As the residual stresses in the simulations vary over short distances on the surface the stresses over the thickness is the mean value of three different locations. A similar behavior is observed for V2 and V3 where $\sigma_{z}$ becomes lower below the feeder on the inner surface. In the case of V1, this difference is less and besides, the behavior is the opposite as $\sigma_{\mathrm{z}}$ is lower between the feeders.
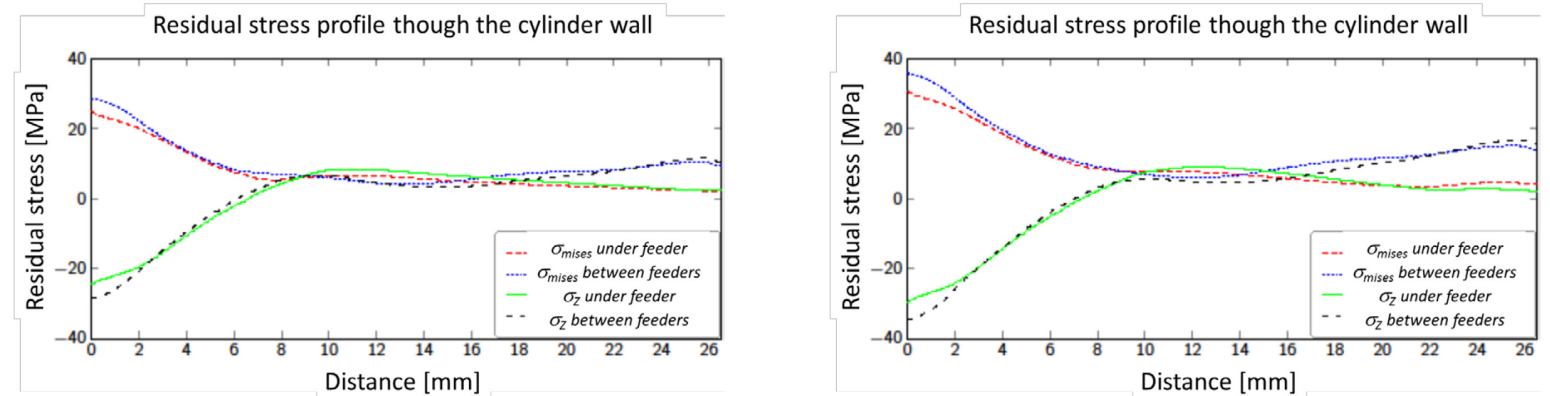

Figure 6. Residual stress profile through the cylinder wall for V2 and V3. Distance equal to zero is the outer surface of the cylinder.

This depends most probably on the mold in V1 that is assumed to be more rigid than V2 and V3 which gives another behavior. As earlier mentioned, the residual stresses in the feeders were not calculated. They only contributed with their thermal influence to the calculation of the residual stresses in the cylinders. In all simulations there is a variation over a distance of only a few millimeters on the surface of the cylinder. It is also possible to see a pattern in these variations that seems to be independent of other geometries such as feeders and gating system. These patterns can be explained by the geometry of the mesh. The variation appears where the geometry has corners due to the curvature of the cylinder. This geometry dependence is most obvious in version V1 and least obvious in V3 and increases therefore with mold stability. It is unclear how this affects the result and if it makes the result unreliably. One obvious way to solve the problem is to increase the number of elements which should minimize this effect and give a convergence control.

Another problem related to hole-drilling and the simulations is that the measurements show a non-uniform stress state through the depth. This affects the resolution and would need a couple of 
elements per millimeter for a good comparison. This also means that the number of elements needed would be more or less unrealistic.

\section{Summary}

Based on the findings in this work, it can be concluded that:

- The measurements done by the sectioning method gives good agreement with the simulation of $\sigma_{z}$ for versions V1 and V3.

- The heat from the feeders affects the development of residual stresses locally and contributes to differences in stresses beneath the feeders compared to the same area between the feeders.

- In order to get use of measurements done by hole-drilling together with simulations it is necessary to have the same resolution on the mesh as depth of each step in the measurement.

\section{References}

[1] F.A. Kandil, J.D. Lord, A.T. Fry, P.V. Grant, A review of residual stress measurement methods - A guide to technique selection, NPL Report MATC(A)04, ISSN 1473-2734 (2001).

[2] P.J. Withers, H.K.D.H. Bhadeshia, Residual stress Part 1 - Measurement techniques, Mater Sci Tech Ser 17 (2001) 355-365.

[3] N.S. Rossini, M. Dassisti, K.Y. Benyounis, A.G. Olabi, Methods of measuring residual stresses in components, Materials and Design 35 (2012) 572-588.

[4] J. Gibmeier, M. Kornmeier B. Scholtes, Plastic deformation during application of the holedrilling method, Mater Sci Forum 347-349 (2000) 131-137

[5] P.V. Grant, J.D. Lord, P.S. Whitehead, Measurement good practice guide no. 53 - issue 2, The measurement of residual stresses by the incremental hole drilling technique, ISSN 1744-3911, (2006).

[6] Measurement of residual stresses by the hole-drilling strain gage method, tech note tn-503, Tech. rep., VISHAY Precision Group (2010).

[7] G.S. Schajer. Hole-Drilling Residual Stress Measurements at 75: Origins, Advances, Opportunities. Exp Mech 50 (2010) 245-253.

[8] T.V. Rajan, C.P. Sharma, A. Sharma, Heat Treatment: Principles and Techniques, PHI Learning Private Limited, (2011).

[9] Y. Motoyama, Y. Inoue, G. Saito, M. Yoshida, A verification of the thermal stress analysis, including the furan sand mold, used to predict the thermal stress in castings, Journal of Material Processing Technology 213 (2013) 2270-2277. 